\title{
Espazo Compartido: Formación Socio-Laboral para Personas con Discapacidad Cognitiva Leve
}

\author{
Antía Díaz, Eloy Calvo \\ Universidade da Coruña. España
}

\begin{abstract}
Resumen
Espazo Compartido surge como una iniciativa de ayuda a la inclusión social y laboral de personas con discapacidad cognitiva leve, que están interesados/as en mejorar su formación, empleando una metodología adaptada a sus necesidades educativas. Los objetivos principales son: Implementar y evaluar un programa universitario en habilidades socio-laborales para personas con discapacidad cognitiva, contando con una asesoría adecuada. Formar a estas personas en habilidades laborales básicas y favorecer su independencia, orientándolos a las posibilidades del mercado laboral. Evaluar el programa con una investigación que muestre el proceso de inclusión desde su perspectiva y la de sus familias.
\end{abstract}

Palabras clave: discapacidad, inclusión, investigación, formación, empleabilidad.

Espazo Compartido es un programa promovido por la Universidade da Coruña (UDC), de formación en habilidades personales, sociales y competencias básicas, orientado a la mejora de la empleabilidad y el fomento de la inclusión laboral de jóvenes con discapacidad cognitiva.

Se trata de un programa pionero en el sistema universitario gallego y una referencia en el ámbito español, que cubre desde 2013 un vacío formativo, como una alternativa para las personas con discapacidad cognitiva que hayan agotado la edad de escolarización. Se celebra en distintas instalaciones universitarias, principalmente en la Facultade de Ciencias da Educación, Facultade de Dereito, Edificio de Servizos Centrais de Investigación y en el Centro Universitario de Riazor.

Espazo Compartido es un proyecto de colaboración universidad-empresa-tercer sector, impulsado por el Consello Social de la UDC, la Fundación UDC (FUAC) y el Vicerrectorado del Campus de Ferrol y de Responsabilidad Social de la UDC, con la colaboración de la Asociación Down Coruña y la Universidad Andrés Bello de Chile, y con el apoyo y financiación de Santander Universidades y Fundación Universia.

El objetivo principal del programa es incluir en el espacio universitario a personas con discapacidad cognitiva que no consiguen acceder por los cauces ordinarios, utilizando una pedagogía adaptada a sus necesidades e incluyendo una certificación académica. Pretende facilitar que los estudiantes construyan un proyecto de vida que les permita desenvolverse de una forma autónoma y con una autoimagen positiva. $\mathrm{Y}$ fomentar la investigación en torno a la discapacidad cognitiva y la inclusión educativa y laboral de este colectivo.

\section{Método}

Para el acceso al programa formativo es necesario cumplir una serie de requisitos:

- Ser mayor de 18 años. Haber agotado la etapa escolar.

- Tener concedida una calificación de discapacidad cognitiva igual o superior al $33 \%$.

- Demostrar una conducta social ajustada.

- No presentar problemas significativos de salud mental.

- Poseer competencias básicas tales como: lectura, escritura y cálculo. Autonomía personal.

- No haber superado las pruebas de acceso a la universidad.

Además de cumplir con estos requisitos, todos los candidatos deben superar un proceso de selección, en el que participan también las familias.

Las pruebas por las que pasan los interesados comprenden:

- Evaluación psicológica a los candidatos: Se analizan los perfiles psicológicos de los solicitantes. Buscando una conducta social adaptada, niveles de madurez adecuados y descartar problemas significativos de salud mental.

- Evaluación logopédica: Se miden las habilidades comunicativas de los candidatos, así como el dominio del lenguaje de cara a poder seguir la formación adecuadamente.

- Entrevista y evaluación psicológica a las familias: Se transmite la metodología, la necesidad de compromiso y contacto continuo con el equipo docente para la continuidad del trabajo que se realice en el aula, principalmente el relacionado con los procesos de autonomía.

En la segunda edición, se añade una evaluación psicológica básica a las familias y /o responsables de los candidatos

- Competencias básicas: Se estudia el manejo autónomo de herramientas de la vida diaria (cálculo, manejo del reloj,...), así como el nivel de comprensión a la hora de seguir instrucciones.

Con este proceso de selección se trata de asegurar el cumplimento de los requisitos de acceso al curso y confirmar que las personas que entren a formar parte puedan ajustarse al cambio con apoyo desde el contexto familiar además del proporcionado desde la Universidad. 


\section{Participantes}

En la actualidad hay dos promociones de estudiantes, formadas por un total de 23 jóvenes de entre 19 y 36 de años, procedentes de diferentes entidades y centros de estudios de A Coruña, Ferrol, Neda, Betanzos y Carballo. La distribución por sexo responde a un total de 10 mujeres y 13 hombres con residencia en A Coruña, Betanzos, Neda, Carballo y Ferrol.

En la primera edición del Programa superaron las pruebas de selección un total 11 candidatos, formado la primera promoción de estudiantes de Espazo Compartido. La distribución de datos de este primer proceso de selección responde a un total de 13 sujetos, 10 hombres y 4 mujeres de entre 18 y 35 años.

Para la segunda edición, se enfrentaron a las pruebas un total de 17 personas, 7 hombres y 10 mujeres de entre 18 y 31 años de los cuales superaron las pruebas 13 personas.

\section{Primera promoción}

El programa abre su primera edición en octubre de 2013 con una presentación ante la comunidad universitaria en el Rectorado de la Universidad de A Coruña.

Los estudiantes que ingresan en el curso responden al siguiente perfil: 7 alumnos con síndrome de Down, 3 alumnos con trastorno del desarrollo intelectual y 1 alumno con autismo. La edad de los ingresados se encuentra entre los 18 a 34 años. Además de la heterogeneidad del grupo, su situación socio- familiar también varía. En su mayoría, un total de 8 estudiantes, viven en contexto familiar, mientras que los 3 alumnos restantes, viven en un centro en régimen interno (Centro Pai Menni).

Estos alumnos han llegado al Programa desde las asociaciones de Down Coruña, Teima-Down Ferrol y Centro Pai Menni (Betanzos).El grupo de alumnos seleccionado, debido a su heterogeneidad, requiere un período inicial de observación en contexto de aula en el que se detectan las necesidades individualidades y las generadas por la adaptación al nuevo entorno.

Se planifican intervenciones que potencien la consolidación de grupo a través de entrenamiento en habilidades sociales y /o comunicativas. Así como un trabajo de desarrollo personal en todos los casos.

\section{Segunda promoción}

La entrada de la segunda promoción se celebra en la Facultad de Ciencias de la Educación, con la presencia de ambos cursos de estudiantes y sus familias. En el curso académico 2014- 2015 entran un total de 13 estudiantes. Se forma un grupo de 16 alumnos/as contando con tres repetidores del curso anterior.

Los nuevos alumnos/as llegan con diagnósticos diversos respecto a los ingresados en el curso anterior.

Como característica común presentan discapacidad cognitiva, pero esta vez, asociada a múltiples patologías y trastornos:

- Hernia de Arnold-Chiari.

- Retraso en el desarrollo psicomotor.

- Incontinencia pigmentaria.

- $\quad$ Síndrome de Di George.
- Heterotopía modular peri ventricular.

- Trastorno Déficit de Atención e Hiperactividad.

- Retraso madurativo por etiología desconocida.

- $\quad$ Retraso oculomotor por presión en el parto.

- $\quad$ Síndrome de West.

- Trastorno del Desarrollo Intelectual

- $\quad$ Síndrome de Down

- Trastorno de Espectro Autista.

El nuevo grupo de estudiantes presenta por tanto, el siguiente perfil: 2 alumnos/as con síndrome de Down, 10 alumnos/as con trastorno del desarrollo intelectual y 1 alumna con autismo. En este caso, todos los estudiantes viven en contexto familiar

Se requiere de nuevo, un período inicial de observación en el que se detectan las necesidades individualidades y las generadas por la adaptación al nuevo entorno. Es importante la observación de las relaciones sociales con los compañeros del programa y alumnos oficiales de la Universidad de A Coruña. El grupo de alumnos/as es mayor y por tanto también su visualización en el entorno.

\section{Equipo Docente}

Para la implementación del programa se cuenta con un equipo multidisciplinar que desarrolla el programa, con diferentes visiones, formación y experiencias. Está formado por dos psicopedagogos, una logopeda, una educadora social, dos psicólogos y un sociólogo. Todos con formación especializada y experiencia en el ámbito de la discapacidad y la educación. Esto se convierte en una fortaleza, ya que todos los miembros se nutren del conocimiento de cada uno de los compañeros.

Por las características del colectivo al que va dirigido, un factor importante para el profesional, es la motivación hacia el trabajo con estas personas. No desde la óptica del asistencialismo a alguien que está en una situación desfavorecida, sino desde el convencimiento de que estas personas pueden mejorar su calidad de vida. Para ello se necesita una alta capacidad de empatía y sensibilidad hacia las problemáticas que cada alumno/a presenta.

Es necesario un equipo interdisciplinar como este que permita implantar la metodología adecuada.

\section{Metodología en el aula}

La educación superior como campo de intervención dirigido a las personas con discapacidad cognitiva es un terreno poco explorado, Partiendo de la premisa de que siempre se pueden encontrar barreras en espacios de nueva intervención, la universidad no es una excepción. La metodología empleada, con dos docentes presentes en el aula, favorece una mayor atención al alumnado y la evolución del grupo, teniendo en cuenta los distintos ritmos de aprendizaje. Junto con esto, se plantea un trabajo teórico - práctico centrado en la persona, con un acompañamiento permanente. Considerando su historia de vida, situación presente y motivaciones personales, se fomenta la construcción de un proyecto de vida (identidad y autonomía). Para favorecer la autoestima, se lleva a cabo un trabajo práctico y reflexivo en torno a la auto-imagen y al auto-conocimiento de los/as participantes. 
La inclusión de las personas con discapacidad en el espacio universitario se afronta desde una perspectiva amplia, considerando la estructura universitaria como elemento clave (relaciones con el personal administrativo, otros alumnos y docentes, utilización de los servicios de la universidad, participación en actividades universitarias, etc.). Así como también observando el rol que debe jugar la familia (tanto para la inclusión universitaria como laboral) y el mercado laboral (capacidad instalada a nivel empresarial para la integración laboral de personas con discapacidad).

Para la evaluación de actividades en el aula, los estudiantes se enfrentan a exámenes y entregas de trabajos, tanto grupales como individuales. Además se realiza un seguimiento académico y personal constante. Los docentes establecen criterios comunes a todas las asignaturas, para después pautar la realización y entrega de tareas, participación en el aula e iniciativa de los estudiantes en cada uno de los módulos, buscando en todo momento potenciar la comunicación entre profesoralumno, así como el aprendizaje cooperativo del grupo.

\section{Estructura del curso}

Espazo Compartido se estructura en 3 cursos de dos cuatrimestres con sus respectivas evaluaciones. El desarrollo de las materias es progresivo es cuanto a grado de dificultad y especialización hacia el mundo laboral. Se trata de que los participantes encuentren relación entre sus aprendizajes personales y los aprendizajes técnicos.

El primer año de estudios se centra en materias que les ayude en su proceso de adaptación al entorno así como el fomento hábitos de estudios estableciendo pautas acordadas con el contexto familiar. En el primer cuatrimestre a través de las materias de Inclusión Universitaria, Desarrollo Personal I, Habilidades Comunicativas I y Reflexión y Autoaprendizaje I se les acompaña en su llegada a la Universidade da Coruña. El segundo cuatrimestre se orienta el aprendizaje de materias técnicas que les proporcionan los conocimientos base para poder dirigir sus estudios, en los siguientes cursos, hacia la adquisición y mejora de habilidades básicas para el empleo a través de materias como Preparación para el Empleo, Matemáticas para el Empleo y Nuevas Tecnologías. Según el curso en el que se encuentren, las materias se especializan en niveles de dificultad.

Así mismo, se crea el programa de prácticas formativas para los estudiantes de Espazo Compartido. Se divide en dos fases claramente diferenciadas. Por una parte, los alumnos/as que llegan a segundo curso realizan prácticas internas, es decir, en diferentes servicios universitarios durante un total de 80 horas en las que se produce, para muchos/as, su primer acercamiento al mundo laboral. En tercer curso ejecutan sus "Primeras Experiencias Laborales" en empresas externas. Se plantean retos que parten de la necesidad de destacar la capacidad productiva de las personas con discapacidad cognitiva dentro del tejido empresarial así como mejorar la empleabilidad y fomentar la inclusión laboral de los jóvenes con discapacidad cognitiva. El Programa de
Prácticas permite a los alumnos tener una experiencia profesional real repartida en otras 80 horas de actividad.

Los participantes (alumnado y sus familias) disponen de servicios adheridos al programa para afianzar todos los procesos que se lleven a cabo dentro y fuera del aula. Se trata de una red de apoyos organizada en función de las necesidades individuales de los estudiantes.

La presencia de dos promociones en el entorno universitario requiere de un periodo de observación importante. Se produce por vez primera la convivencia de dos generaciones de estudiantes con discapacidad cognitiva. Para poder extender la intervención a su vida cotidiana se trabaja con cada uno de los participantes y sus familias, a través de diferentes servicios:

- Servicio de Atención a Familias: Se establece en cada caso un plan de trabajo que complemente las acciones del aula para favorecer la autonomía y desarrollo personal en el contexto familiar.

La transversalidad de objetivos y trabajo conjunto con el vínculo directo de los estudiantes es básica para la generalización de aprendizaje.

-Servicio de Apoyo Psicológico: Seguimiento de la evolución de cada alumno acompañamientos en el proceso de desarrollo madurativo y personal en contexto universitario. El equipo de apoyo psicológico transfiere la información obtenida en estas sesiones favoreciendo el desarrollo personal y el cumplimiento de objetivos a través de la planificación académica y planes individuales de los alumnos

-Servicio de Refuerzo Académico: Se establece un plan de trabajo común adaptado a las necesidades individuales. En horas extraacadémicas se imparten horas de refuerzo donde se enseñan técnicas de estudio y se favorece la rutina de trabajo. Dado que se realiza en el Centro Universitario de Riazor con estas actividades se busca que los alumnos interactúen en el entorno fomentando la vida universitaria como parte del proceso de inclusión.

-Servicio de Comunicación con Profesionales Externos: Se crea una red de contactos y comunicación sólida con los diferentes profesionales que intervienen en el desarrollo madurativo de cada uno de los alumnos. Abrimos este Servicio tras la valoración de una necesidad en la coordinación de objetivos y método de trabajo con todos aquellos que intervienen en la Calidad de Vida del alumno.

-Servicio de Tutorías de Preparación para el Empleo: Partiendo de uno de los objetivos principales del programa, favorecer la empleabilidad de las personas con discapacidad cognitiva, y la implementación del programa de prácticas, se articula un servicio de apoyo para el ajuste de aptitudes, actitudes enfocadas al empleo.

\section{Investigación}

Teniendo en cuenta que es un proyecto pionero a nivel autonómico, es preciso medir su impacto y presentar los resultados al resto de la comunidad científica de cara a abordar la educación superior de las personas mayores de 18 años con discapacidad cognitiva. Lo que se pretende al fin y al cabo, es formar y ayudar a 
desarrollar los alumnos/as en personas con valores y capacidades que les permitan desenvolver actividades que se retroalimenten en la misma sociedad como también en ellos mismos como individuos.

Por este motivo, la investigación que se lleva a cabo, busca a través de un método mixto (cuantitativo/ cualitativo) conocer los cambios que han experimentado los estudiantes y sus respectivas familias durante su estancia en el contexto universitario.

Se procede a la aplicación de cuestionarios elaborados a partir de los diferentes procesos del programa (contenidos en el aula, atención a familias, procesos administrativos, infraestructura...) que permiten a los alumnos y familiares responder y así, conocer su visión global respecto a los procesos llevados a cabo por el programa y cómo repercute en su desarrollo. Por otro lado, se realizan "grupos de conversación" en dónde se analiza de forma conjunta los avances comunes de los estudiantes, los cambios en la rutina familiar y la evolución del alumno/a en su rol adulto.

De los resultados provisionales obtenidos en el curso académico 2013-2014, se observa cómo los propios alumnos y sus familias reconocen sus aprendizajes y a nivel general valoran el programa satisfactoriamente.

En la Figura 1 podemos ver como los estudiantes identifican que el programa es útil para introducirse en el mercado laboral, con lo que reconocen de forma implícita que les favorece en su proceso de madurez. Para los estudiantes en general, ha sido la primera vez que han realizado trabajos en grupo, y se puede observar en sus valoraciones que es una actividad que les satisface. También se manifiesta su agrado con sus profesores y compañeros, lo cual favorece, entre otros, su valoración respecto a los aprendizajes en materia de comunicación y desarrollo personal. Se evidencia un enriquecimiento en las relaciones sociales y el aprendizaje en la utilización de los servicios universitarios. Todos los ítems son valorados con puntuaciones cercanas a 5 , indicando que están bastante satisfechos con sus aprendizajes en contexto universitario.

Figura 1. Resultados de aprendizaje alumnos 1

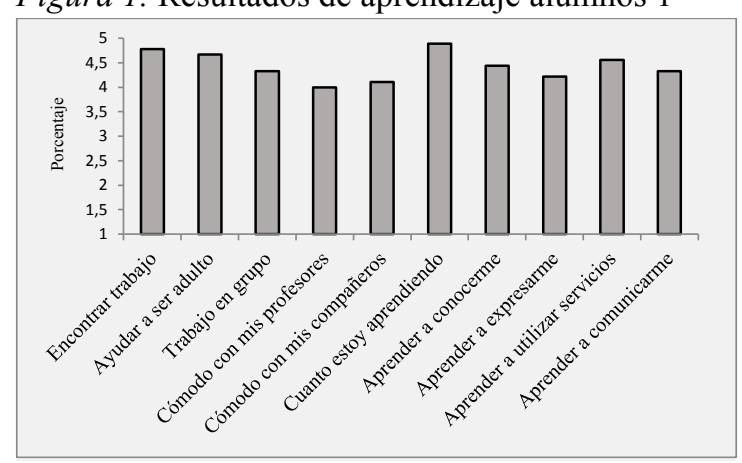

Tal y como se puede observar en la Figura2. Resultados de aprendizaje alumnos 2, los estudiantes valoran con puntuaciones cercanas a 10, los temas de las asignaturas, las materias del segundo cuatrimestre del primer curso, las tutorías con los profesores, la Universidad y el programa en general. Esto deja entrever que los estudiantes se sienten bastante satisfechos en esta nueva etapa formativa y que los aprendizajes interiorizados hasta el momento dan sus frutos, tal y como se puede observar en la figura anterior.

Figura 2. Resultados de aprendizaje alumnos 2

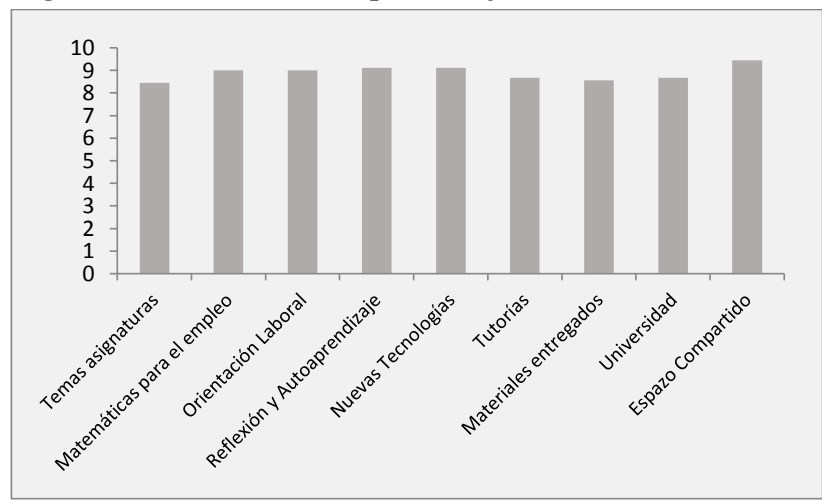

Las familias participantes en el programa reconocen, tal como se ve en la Figura 3. Resultados de aprendizaje, que sus respectivos hijos/as estudian materias idóneas para su desarrollo, así como una evolución en su participación en contexto familiar, desarrollo de la capacidad crítica, habilidades comunicativas, motivación personal y resolución de conflictos. En materia de destrezas técnicas orientadas al mundo laboral, indican una valoración muy positiva, reconociendo una mejoría en cuanto al manejo de ordenador o técnicas de búsqueda de empleo entre otras.

Figura 3. Resultados de aprendizaje familias

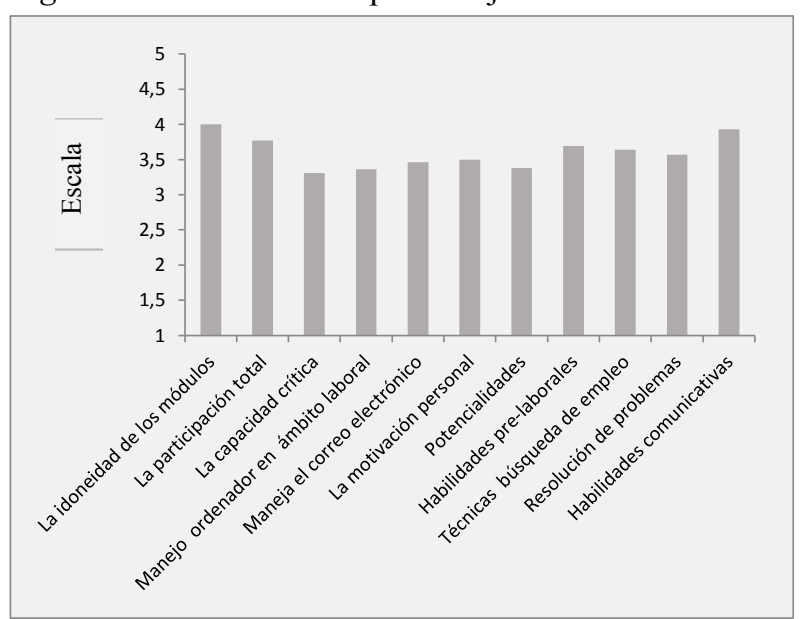

Figura 4. Satisfacción general familias

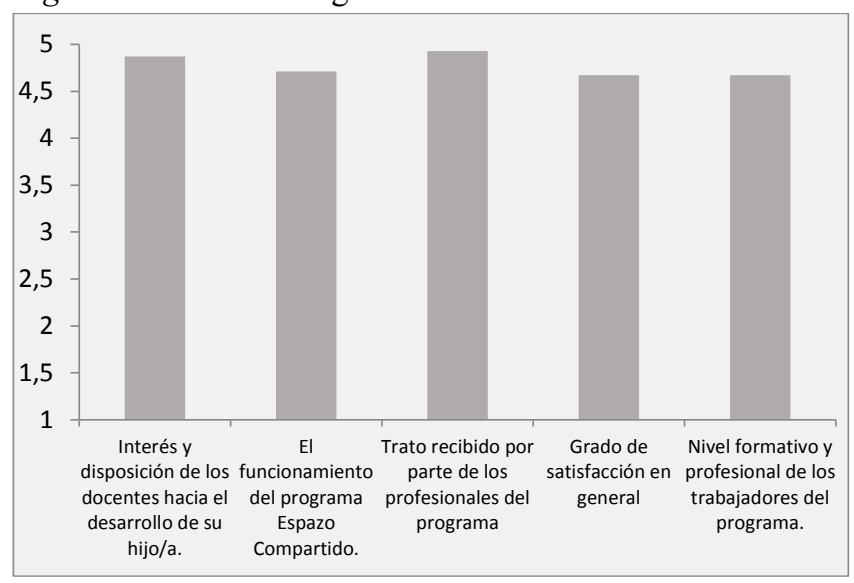


Los datos reflejan una percepción positiva en cuanto al desarrollo madurativo de sus hijos. Esto hace que las familias valoren con puntaciones de 5 y cercanas a $5 \mathrm{su}$ grado de satisfacción con el programa tal y como muestra la Figura 4. Valoración general del programa. Las familias destacan el trato recibido, grado de formación e interés y disposición de los profesionales del programa. Por tanto, la valoración respecto al funcionamiento y grado de satisfacción general responden a puntuaciones altamente satisfactorias. 